\title{
THEORETICAL ANALYSIS OF PROCESSES IN AUTOMATIC CONTROL SYSTEM
}

\author{
Galina Starodubtseva, Sergey Livinsky, Elena Rubtsova, Svetlana Lyubaya \\ Stavropol State Agrarian University, Russia \\ sgp_starodubtseva@mail.ru, lis-nc@yandex.ru, elen.68rubtsova@yandex.ru, unil.sgau@yandex.ru
}

\begin{abstract}
The theoretical analysis of processes in the system "voltage converter - working chamber - layer of seeds" is carried out in the article. The system "voltage converter - working chamber - seed layer" is presented as a continuous link of the automatic control system. The principle of operation of the network voltage converter for the plant for presowing seed treatment by a pulsed electric field is created with the purpose of increasing their seeding qualities and suppressing the pathogenic microflora is presented. One of the components of the proposed device is an alternating current (AC) voltage regulator, used to solve power problems from a rural power grid. The voltage stabilizer is made in autotransformer switching, and the location of the windings is such that the stabilization of the voltage on the load, when changing both the mains voltage and the magnitude of the load, is performed smoothly in the entire range without interrupting the mains and load circuits. Advantages of the stabilizer: a wide range of voltage stabilization; high stability of the voltage on the load; smooth, stepless regulation and stabilization of output voltage; Absence of switching overvoltages and inrush currents. The proposed device differs from existing ones because due to the constant monitoring of energy processes in the layer of processed seeds, and feedback to the control unit, the transformation of the parameters of the pulsed electric field is automatically provided. The automatic transformation of the electric field parameters depending on the changes that have occurred in the seed layer, using the example of seeds with different humidity, is able to maintain the required treatment dose, ensuring stability and repeatability of the results. Synthesis of the structural and circuit diagrams of the voltage converter for presowing seed treatment and its control scheme is performed. The structural scheme of the converter and the principle of its operation are considered.
\end{abstract}

Keywords: automatic control, network voltage converter, pulsed electric field.

\section{Introduction}

Presowing processing of crops with environmentally friendly methods to improve their sowing qualities is becoming increasingly important due to the growing environmental pollution with pesticides used in agriculture for many decades. This conclusion is confirmed by the studies of a number of scientists, who made presowing treatment with physical factors.

Processing of seeds of agricultural crops by a pulsed electric field, as the results of researches show, gives good results in the fight against contamination of seeds with pathogenic mycoflora, increase in germination energy and germination of seeds [1-3]. Presowing treatment of soybean seeds with a pulsed electric field was winter wheat [4], carried out [5-6], onion seeds [7-8], beans and corn [9-10], cotton seeds [11] and etc. In laboratory and field experiments an increase in seed germination to $12 \%$ was obtained; productivity up to $15 \%[12 ; 13]$.

Introduction of pulsed electric field (IEP) seed treatment in the technological process of seed preparation for sowing or seed processing, is blocked by insufficient research on rational regimes for seed treatment of various crops, as well as lack of understanding of influence of physical factors on biological objects.

In the IEP seed processing plants studied, the operator manually sets the mode for the crop to be treated, and then manually changes the treatment mode, depending on the degree of humidity, trauma and the size of the seed being treated. For each batch of seeds, a series of laboratory experiments is carried out before processing to select treatment regimes. As a consequence, weak stability and repeatability of the results obtained is observed, as well as complete lack of automatism.

In industrial (in-line) processing, the load is a layer of seeds having various degrees of contamination, injuries, humidity and seed sizes. Therefore, a plant is needed that makes it possible to determine such changes in the mass to be processed and automatically convert the parameters of the effect: amplitude, duration and pulse repetition frequency, depending on the changes that have occurred.

To solve the above problems we have developed the electrical equipment of the plant, the theoretical analysis of processes in the automatic control system of which is proposed in this article. 


\section{Materials and methods}

To fulfil our goal, we conducted a theoretical analysis of processes in the system "voltage converter - working chamber - layer of seeds." The system "voltage converter - working chamber - a layer of seeds" is represented by us as a continuous link of the automatic control system.

$$
G=\frac{D_{1}^{2}}{f 4 \tau}\left(\sqrt{1+\frac{f 8 \tau}{D_{1}^{2}}-1}\right),
$$

where $f$-conversion frequency, $\mathrm{kHz}$;

$D_{1}-$ converter duty ratio pulse repetition;

$\tau$ - conversion period, $\mu$ s.

$$
D_{1}=G \sqrt{\frac{f 2 \tau}{1-M}},
$$

where $G-$ regulator voltage transfer ratio from input to DC output.

$$
\left\{\begin{array}{l}
-\frac{\sum D}{L} U_{C}+\frac{d_{1}}{L} u_{i n}=0 \\
\hat{u}_{c}=\frac{\sum d}{C} i_{L}-\frac{1}{R C} u_{c}
\end{array},\right.
$$

where $i_{L}$ - instantaneous current in inductance;

$u_{\text {in }}$ - instantaneous processing stress level;

$u_{c}$ - instantaneous stress on the capacity of the seed layer;

$U_{c}$ - effective value stress on the capacity of the seed layer;

$R$ - load resistance (working chamber with loaded seeds);

$C$ - load capacity (working chamber with loaded seeds);

sum $(d) ; \operatorname{sum}(D)$.

The requirements to the plant stabilizer for presowing seed treatment are formulated:

1. smooth, stepless regulation and stabilization of output voltage;

2. elimination of switching interruptions in the power supply of the installation;

3. elimination of switching overvoltages and inrush currents;

4. high efficiency;

5. compactness;

6. affordable price.

\section{Results and discussion}

At present, the load on the rural grid is increasing. And the more acute problems of the rural power grid are becoming more acute: the remoteness of the substation or transformer, the heterogeneity and poor throughput of the cable network, etc. Besides, there can be no network at all, and the equipment can be operated from an electric power unit. And the nature of the load, when processing the grain mass, is capacitive and the current consumed from the source does not coincide in phase with the voltage. Consequently, the quality of electricity consumption from the network will be low. In the absence of a network, the electrical generator will have to select an overestimated power relative to the load. Solving similar problems will allow the use of the regulator of the AC voltage regulator.

Such requirements led to the idea of abandoning both of the most common ways of maintaining the output voltage of the stabilizer. Namely, by switching the tapping of the autotransformer winding by a thyristor switch and switching the secondary winding electromechanically.

Let us consider in detail the proposed stabilizer (Figure 1). The stabilizer - the AC voltage regulator consists of the autotransformer 1 on the cores (magnetic circuits) 2 and 3, on which the primary windings 4, 5 and the secondary winding 6 are wound. In addition, the control windings 7 and 8 are wound on the cores of the autotransformer, with the connected blocks of electronic regulators 9 , 
10. The stabilizer is controlled by the control unit 11 , via the feedback circuit 12 , and the potentiometer 13. The output of the stabilizer-AC voltage regulator is connected to load 14. It should be noted that the primary windings 4 and 5 are interconnected in series in opposition.

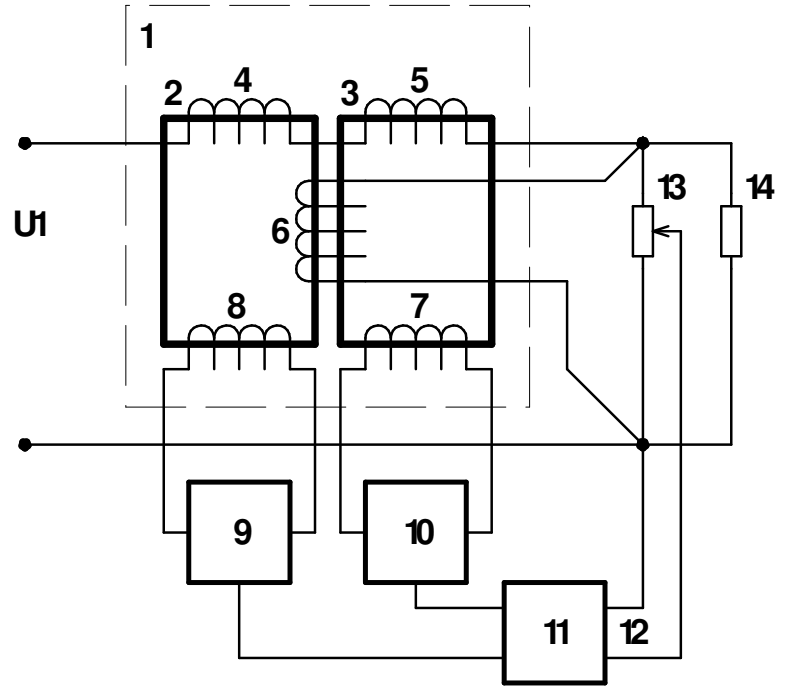

Fig. 1. Functional diagram of stabilizer of regulator of alternating current voltage

The stabilizer works as follows. When connected to an electrical network under the influence of the supply voltage $U 1$, electric current passes through the series circuit of the primary windings 4,5 and the secondary winding 6 of the autotransformer 1 . This current creates magnetic fluxes in the cores 2 and 3. The total magnetic flux of the cores 2 and 3 is determined by the value of the supply voltage $U 1$. The magnetic fluxes of the cores 2 and 3 , respectively, in the primary windings 4,5 induce the voltage $U 4, U 5$. In the control windings 7,8 , voltages are also induced. The voltage $U 4$ of the primary winding 4 coincides in direction with the supply voltage $U 1$, and the voltage $U 5$ of the primary winding 5 is opposite in direction. When the magnetic fluxes in the cores 2,3 and the same number of turns in the primary windings 4 and 5 are equal, the voltages $U 4$ and $U 5$ are equal in magnitude and opposite in direction.

Therefore, the sum of the voltages $(U 4+U 5)$ at the ends of the series circuit of the primary windings 4 and 5 is zero because of the counter-switching of the windings 4,5 . As a result, the voltage of the secondary winding $U 6$ equal to the grid voltage $(U 3=U 1)$ is applied to the load 14 . This mode of operation of the stabilizer corresponds to the rated voltage in the network $\left(U 1=U_{\text {nom }}\right)$. The voltage diagram for the voltage regulator elements for this mode is shown in Figure 2 (a).

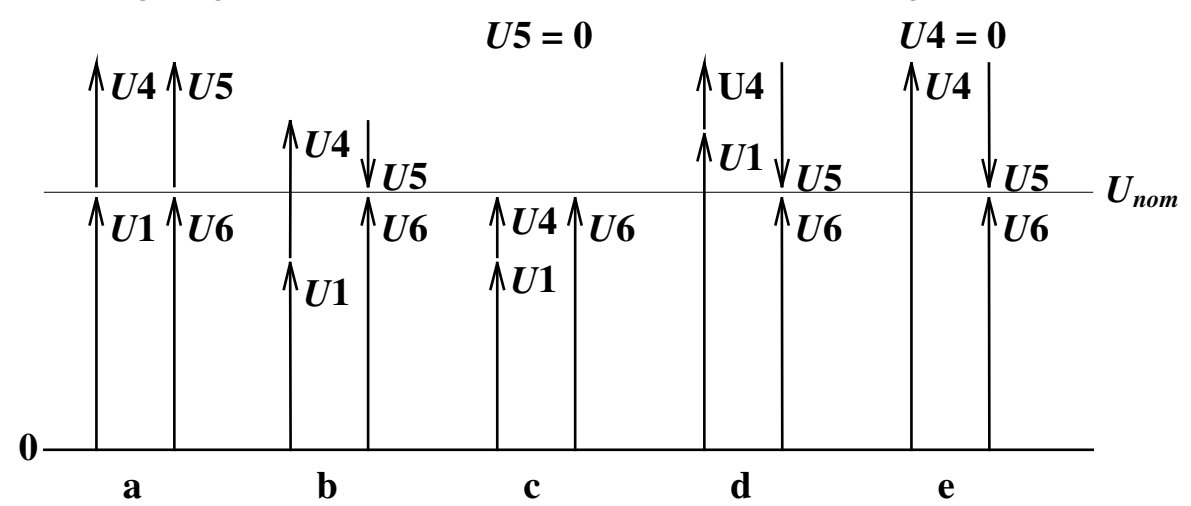

Fig. 2. Diagrams of voltage changes on elements of stabilizer

The control circuit 11, in this mode, commands the blocks 9, 10 of the electronic regulators to establish the greatest resistance (infinite, i.e., an open circuit). The currents in the control windings 7 , 8 are equal to zero, therefore, there is no demagnetization of the cores 2,3 . The idling current in the primary windings 4 and 5 of the autotransformer 1 at the rated voltage of the network $U 1$ is minimal, since the magnetic flux passes through the two cores 2 and 3, the reactive power consumed by the stabilizer from the network is minimal. When the voltage of the network $U 1$ decreases (and 
accordingly decreases the voltage $U 6$ to the secondary winding 6 and the load 14), a signal of a reduced value for the control system 11 is formed along the feedback circuit 12 . At the command of the control unit 11, the electronic regulator unit 10 decreases the resistance value. The resistance of the electronic regulator unit 9 remains unchanged large. In the control winding 7 of the electronic regulator closed on the resistance 10, a current produces an opposing magnetic flux that reduces the magnetic flux in the core 3. Due to the condition of the invariance of the magnitude of the total flux in both cores 2 and 3, respectively, in the core 2, the magnetic flux increases due to a forced decrease magnetic flux in the core 3 . In the primary windings 4 and 5, there is also an opposite voltage change. Voltage $U 4$ increases, and $U 5$ decreases, the sum of these voltages is not zero $(U 4+U 5>0)$ and is allocated at the ends of the primary winding chain 4,5 . The sum of the voltages $U 4$ and $U 5$, coincides in phase with the mains voltage $U 1$ and adds to it, compensating for its decrease. This ensures stability of the voltage on the secondary winding $6\left(U 6=U_{\text {nom }}\right)$ and load $14\left(U 1+(U 4+U 5)=U 6=U_{\text {nom }}\right)$. This mode corresponds to the stress diagram in Fig. 2 (b). The transition of the stabilizer to a lowered mains voltage occurred smoothly without switching in power circuits, both power supply and load, that is, without power interruptions, without switching and without distortion of the sinusoidal voltage form. When the voltage of the mains $U 1$ decreases, the resistance of the electronic regulator unit 10 decreases to zero. In this case, the control winding 7 becomes shorted. The magnetic flux in the core 3 becomes zero due to the short-circuited state of the control winding 7 . Here, the voltage U5 of the primary winding 5 is zero.

All the total magnetic flux passes through the core 2, the maximum increase in the voltage of the network $U 1$ is created only by the voltage $U 4$ of the primary winding 4 . The sum of the reduced voltage in the network $U 1$ and the voltage of the primary winding $4\left(U 1+U 4=U 6=U_{\text {nom }}\right)$ provides a stable voltage $U 6=U_{\text {nom }}$ on the secondary winding 6 and load 14 (Figure 2 (c)). As the voltage of the network $U 1$ increases, the voltages $U 6$ in the secondary winding 6 and on the load 14 accordingly increase. In this case, the control process proceeds as follows. Through the feedback loop 12 to the control unit 11, a signal proportional to the beginning of the increase in the voltage value $U 6$ is received. At the command of the control unit 11, the resistance value of the electronic regulator unit 9 is reduced.

In this case, the resistance of the electronic regulator unit 10 remains unchanged and has the greatest value (infinity). In the control winding 8 a current that reduces the magnetic flux in the core 2 flows to the control unit of the electronic regulator 8. Due to the condition of the invariance of the magnitude of the total flux in both cores 2 and 3, respectively, in the core 3, the magnetic flux increases due to a forced decrease in the magnetic flux in the core 2 In the primary windings 4 and 5 there is also an opposite change in the voltages. Voltage $U 5$ increases, and $U 4$ decreases, the sum of these voltages is not zero and is allocated at the ends of the secondary winding chain 4,5 . The sum of the voltages $U 4$ and $U 5$ is in antiphase with the mains voltage $U 1$ and is a reducing voltage for it, compensating for its increase. This ensures the stability of the voltage on the secondary winding 6 $\left(U 6=U_{\text {nom }}\right)$ and the load $14\left(U 1-(U 4+U 5)=U 6=U_{\text {nom }}\right)$. This diagram corresponds to the stress diagram in Fig. 2 (d). The transition of the stabilizer to an increased line voltage occurred smoothly without switching in power circuits, both power supply and load, that is, without power interruptions, without switching and without distortion of the sinusoidal voltage form. At the maximum increase of the mains voltage $U 1$, the resistance of the electronic regulator unit 9 and the voltage $U 4$ of the primary winding 4 decreases to zero. In this case, the control winding 8 becomes shorted. The magnetic flux in the core 2 becomes zero due to the short-circuited state of the control winding 8 . In this case, the voltage $U 4$ of the primary winding 4 is zero. All the total magnetic flux passes through the core 3, the maximum reduction in the voltage of the network $U 1$ is created only by the voltage $U 5$ of the primary winding 5 . The difference in the voltages of the network $U 1$ and the voltage of the winding $5\left(U 1-U 5=U 6=U_{n o m}\right)$ provides a stable voltage $U 6=U_{\text {nom }}$ on the secondary winding 6 and load 14. This diagram corresponds to the stress diagram in Figure 2 (e). In real regulators of the mains voltage at a constant voltage of the power supply network $U 1$, and when the value of the load resistance 14 changes, the value of the voltage $U 6$ on the secondary winding 6 of the autotransformer 1 also changes. This is due to a change in the voltage drops on the active and inductive resistances of the stabilizer itself. The proposed device also provides stabilization of the voltage on the load with a change in its magnitude. 
Adjustment of the output voltage on the load 14, regardless of the input voltage, is carried out using a potentiometer 13. By changing the ratio of the resistance of the potentiometer 13, set the desired value of the output voltage. On the feedback circuit 12 to the control unit 11, a signal proportional to the set value of the voltage at the resistance of the potentiometer 13 is received. The control unit 11, by means of the electronic regulator units 9 and 10, adjusts the output voltage. An experimental verification of the operation of the claimed stabilizer voltage regulator was carried out. Core cores of magnetic circuits, rod and ring were used. The experiments fully confirmed the feasibility of the claimed device. From the analysis of the device operation and conducted experiments it follows that the voltage stabilization on the load 6 , when changing both the voltage of the network $U 1$ and the value of the load 14, is made in the entire range smoothly without breaking the mains and load circuits. The magnetizing current is only allowed once, when the stabilizer is turned on.

\section{Conclusions}

The application of the described stabilizer will allow to obtain the following advantages:

1. wide range of voltage stabilization;

2. ensuring a high stability of the voltage on the load;

3. smooth, stepless regulation and stabilization of the output voltage;

4. absence of switching interruptions in the power supply;

5. absence of switching overvoltages and inrush currents.

\section{References}

[1] Bilalis D.J., Katsenios N., Efthimiadou A., Karkanis A. Pulsed electromagnetic field: An organic compatible method to promote plant growth and yield in two corn types, 1983. Electromagnetic Biology and Medicine, 2012, vol. 31(4), pp. 333-343.

[2] Borodin I.F.. Development of electro-technology in farm production. In Mechanization and Electrification of Agriculture, no. 6, pp. 27-31.

[3] Dymek K., Dejmek P., Panarese V., Vicente A.A., Wadsö , L. Finnie, C., Galindo, F.G. Effect of pulsed electric field on the germination of barley seeds, LWT, Food Sci. Technol, 47, 2012,. pp. 161-166.

[4] Livinskiy S.A., Starodubtseva G.P., Afanasev M.A. Voltage transformer for the installation of pre-sowing seed treatment. In APK Stavropolie. Bulletin, 2016, no. 4, pp. 35-39.

[5] Hainovskiy V.I. Starodubtseva G.P. Rubtsova E.I. Hachenko A.A. The method of pre-sowing treatment of soybean seeds by electric field. Patent for invention RUS 2340139, 02.04.2007.

[6] Hainovskiy V.I. Starodubtseva G.P. Rubtsova E.I. Pre-sowing stimulation of seeds of soybean pulsed electric field. In Mechanization and Electrification of Agriculture. 2007, no. 10, pp 17-18.

[7] Hainivskiy V.I., Lubaya S.I., Kopulova O.S., Afanasev M.A. The method of pre-sowing seed treatment. In Machine Operator. - 2017, no. 1, pp. 14-15.

[8] Hnukina A.G. The rationale of the electro-technological parameters and modes of the low-voltage activator for pre-sowing treatment of onion seeds: dis. cand. tech. sciences: 05.20 .02 / Hnukina Anna Georgievna; - Stavropol, 2014, 169 p.

[9] Nelson S.O., Guo W., Trabelsi S., Kay S.J. Dielectric spectroscope of watermelons for sensing quality. In Measurement Science and Technology, 2007, vol.18, pp. 1887-1892.

[10] Novák J., Vitázek I., Electrical properties of sunflower achenes. In Acta Technologica Agriculturae. 2014, no.4, pp. 109-113.

[11] Yan Y., Zhou S., Song Z., (...), Zhang X., Song H. Effects of frequency and voltage of high voltage pulsed electric field on improving vigor of aged cotton seed. Nongye Gongcheng Xuebao/Transactions of the Chinese Society of Agricultural Engineering, 2010, pp. 1255-1260.

[12] PATENT 2181234, The Russian Federation, MPK A 01 C1 / 00 The machine for pre-sowing seed treatment in electric field /SHMIGEL, V.V. - NIYAZOV, A.M.; patentee and holder:The Kostroma State Agricultural Academy. -No. 99118792/13; applic. 30.08.99; publ. 20.04.02. Bul. no.11.

[13]PATENT 2412574, The Russian Federation, MPK A 01 S1/00 The method and installation for pre-sowing stimulating and disinfecting seed treatment / V.G. SPIROV: a patent holder of LLC «NPP INTEX» (RU).- № 2009117944/10; applic. 14.05.2009; publ. 20.11.2010. Bul. no.6. 